\title{
Association of Serum Uric Acid Concentration and Its Change with Cardiovascular Death and All-Cause Mortality
}

\author{
Ze-Xuan Dong $\left(\mathbb{D},{ }^{1}\right.$ Ming Tian $\left(\mathbb{D},{ }^{2}\right.$ Hua Li ${ }^{\circ},{ }^{2}$ Yang Wu $\mathbb{D}^{2},{ }^{2}$ Xing-Guo Du $\mathbb{D}^{2},{ }^{2}$ \\ Jun-Wu Dong $\mathbb{D},{ }^{2}$ Hui-Hui Xiao $\mathbb{C}^{3},{ }^{3}$ Li-Ping Dong $\mathbb{C}^{2},{ }^{2}$ and Xiao-Hong Song $\mathbb{D}^{2}$ \\ ${ }^{1}$ Shanxi Medical University, Jinzhong, 030600 Shanxi, China \\ ${ }^{2}$ Department of Nephrology, Wuhan Fourth Hospital, Puai Hospital, Tongji Medical College, Huazhong University of Science \\ and Technology, Wuhan, 430030 Hubei, China \\ ${ }^{3}$ Department of Internal Medicine, JiangHan University, Wuhan, 430030 Hubei, China
}

Correspondence should be addressed to Li-Ping Dong; zdhping@sina.com and Xiao-Hong Song; xiaohongsong1638@163.com

Received 1 September 2019; Revised 29 November 2019; Accepted 23 December 2019; Published 29 January 2020

Academic Editor: Paulina Dumnicka

Copyright (C) 2020 Ze-Xuan Dong et al. This is an open access article distributed under the Creative Commons Attribution License, which permits unrestricted use, distribution, and reproduction in any medium, provided the original work is properly cited.

Objective. There is no consensus on the role of abnormal uric acid (UA) levels in the prognosis of patients undergoing hemodialysis We therefore aimed to investigate the effects of changes in UA concentration on the risk of all-cause death and cardiac death in such patients. Method. In this retrospective cohort study, patients admitted to two hemodialysis centers performing maintenance hemodialysis (MHD) in Wuhan First Hospital and Fourth Hospital Hemodialysis Center from January 1, 2007, to October 31, 2017, were included. Results. In all, 325 patients undergoing MHD aged $59.7 \pm 14.7$ years, including 195 men (60\%), were enrolled, with a median follow-up of 37 months. Serum UA $(p<0.001)$ was significantly higher in the surviving group than in the death group. No significant difference was found in UA variability $(p=0.193)$ between the two groups. Patients were assigned Quartile 1-Quartile 4 (from lowest to highest) based on serum UA levels. A total of 45 of 81 (55.6\%) patients in Quartile 1 experienced all-cause death; 19 of 82 (23.2\%) in Quartile 4 had all-cause mortality (OR, 0.2; 95\% confidence interval, 0.1 to 0.5 ; p for trend < 0.001$) .25$ of $81(30.9 \%)$ in Quartile 1 had cardiac death; and 11 of $82(13.4 \%)$ in Quartile 4 had cardiac death (OR, 0.3 ; $95 \%$ confidence interval, 0.2 to 0.8 ; $p$ for trend $=0.003)$. After adjusting for age, primary renal disease, vascular access, serum albumin, and UA variability, the OR values and 95\% CI for all-cause death and cardiac death were 0.3 and 0.1-0.7 ( $p=0.001)$ and 0.6 and $0.2-1.4(p=0.101)$, respectively. Additionally, the receiver operating characteristic curve showed that UA concentration and its changes combined with age had a good predictive value for all-cause mortality in patients undergoing MHD (AUC $=0.7517,95 \%$ CI: 0.6677-0.811, $p=0.0245$ ). However, the predictive value of cardiac death was not improved (AUC $=0.7394,95 \% \mathrm{CI}: 0.6677-0.811, p=0.2194)$. Conclusion. Low UA levels were closely related to all-cause mortality in patients undergoing MHD. Although UA levels had no significant effect on cardiac death, they had a good predictive value for long-term prognosis in patients on MHD.

\section{Introduction}

Studies have shown that hyperuricemia has a prevalence of more than $20 \%$ in the general population [1]. However, in patients with end-stage renal disease (ESRD), due to renal failure, glomerular filtration rate is severely reduced. The reabsorption of uric acid (UA) increases and secretion decreases in the proximal tubules. This causes a significant decrease in UA excretion, and excessive retention of UA in the body causes hyperuricemia [2]. Therefore, the incidence of hyperuricemia is higher in patients with ESRD, even more than $50 \%$ [3]. However, the average serum UA clearance per hemodialysis is about $1 \mathrm{~g}$. Therefore, for hemodialysis patients, the UA level varies greatly [4].

It is well-known that vascular calcification, especially coronary artery calcification, is ubiquitous in patients undergoing hemodialysis. However, vascular calcification is an important cause of cardiovascular disease occurrence and death. The current etiology and pathogenesis of coronary calcification in patients with ESRD have not been fully 
elucidated. In recent years, many studies have shown that hyperuricemia is another common independent risk factor of vascular calcification, including dyslipidemia, smoking, hypertension, diabetes, and chronic kidney disease (CKD) $[5,6]$. Higher levels of the serum UA led to wider coronary lesions, while heavier stenosis led to higher mortality rates [7-9]. Apart from vascular calcification, the effect of hyperuricemia on patients is also closely related to patients with hypertension, congestive heart failure, and atherosclerosis [10]. Therefore, Europe launched a consensus on the diagnosis and treatment of patients with high uric acidemia and high cardiovascular risk in 2018. It is mentioned in the consensus that serum UA should be controlled below $360 \mu \mathrm{mol} / \mathrm{L}$ in all populations. However, for patients with a high risk of cardiovascular disease, serum UA should be controlled below $300 \mu \mathrm{mol} / \mathrm{L}$ [11]. In fact, whether hyperuricemia is a risk factor for all-cause death and cardiac death in patients undergoing maintenance hemodialysis (MHD) has been a controversial topic. The early studies have found a "J"-shaped relationship between serum UA and all-cause mortality and cardiac mortality in patients with advanced CKD [12]. However, subsequent research has raised contradictory points: the increase in UA levels increased the risk of death in patients undergoing hemodialysis or peritoneal dialysis [13, 14]; in contrast, the lower levels of serum UA resulted in higher risks of all-cause death and cardiac death in patients undergoing hemodialysis $[15,16]$. As time progresses, longitudinal increases in serum UA levels are associated with a reduction in all-cause mortality or cardiovascular death in these patients [4].

Therefore, the current evidence of UA level for all-cause death and cardiovascular death in hemodialysis patients is contradictory, and there is no consensus. This study is aimed at analyzing the effects of serum UA levels and their variability in all-cause death and cardiac death in patients undergoing MHD through a retrospective cohort study.

\section{Materials and Methods}

2.1. Participants. A retrospective cohort study was conducted to analyze the data of patients who underwent MHD in Wuhan First Hospital or Fourth Hospital Hemodialysis Center from January 1, 2007, to October 31, 2017. The inclusion criteria were as follows: (1) stable patients (dialysis vintage $\geq 12$ months); (2) the initial dialysis mode was hemodialysis; (3) patients who underwent regular dialysis via their vascular access for 4 hours two or three times per week at a blood flow rate of $230-300 \mathrm{~mL} / \mathrm{min}$ and a dialysis solution flow rate of $500 \mathrm{~mL} / \mathrm{min}$; the dialysis mode was not limited; (4) there were at least three serum UA tests per year, and the interval between two adjacent examinations was more than 2 months; for the frequent examination within 2 months, we took the average value; (5) the age of starting hemodialysis was $\geq 18$ years old. The exclusion criteria were (1) patients with malignant tumor, (2) hemodialysis converted to peritoneal dialysis, and (3) combination of other serious organ diseases which affected the level of UA.

2.2. Method. Baseline clinical data were collected from patients who underwent hemodialysis, including those on primary renal disease, vascular access, hemoglobin, serum calcium phosphorus, albumin, UA, and parathyroid hormone (PTH). Blood specimens for biochemical tests were collected from the vascular access before midweek hemodialysis sessions. They were collected 7:00-8:00 h before morning sessions and 11:00-12:00 h before midday sessions. Patients were grouped according to the quartile of mean serum UA levels.

2.3. Endpoints of the Study. All patients were followed up from MHD treatment to the endpoint events (cardiac death or all-cause death), kidney transplantation, loss of followup, withdrawal from hemodialysis, or arrival of the study deadline (October 31, 2018). The endpoint event was allcause mortality and cardiac death. Cardiac death was defined as death caused by ischemic heart disease, congestive heart failure, fatal arrhythmia, or other unexplained sudden death [17]. The calculation method for UA variability CV (coefficient of variance) is as follows: $\mathrm{CV}=$ (standard deviation/ mean) $\times 100 \%$, which can better reflect the degree of dispersion in the unit mean. This study was approved by the Ethics Committee of the First and Fourth Hospital of Wuhan and followed the ethical principles of the Declaration of Helsinki (http://www.wma.net/en/30publications/ 10policies/b3/index.html).

2.4. Statistics. Categorical and continuous variables are presented as percentage and mean $\pm S D$, respectively, and nonnormal distributions are reported by the median concentration and interquartile range. The chi-square test and Wilcoxon rank sum test were used to compare categorical and continuous variables among groups, respectively. The analysis of linear trends was used to evaluate the association between increasing levels of UA and the risk of all-cause mortality or cardiac death after the patients were divided into quartiles based on the distribution of controls. A KaplanMeier analysis was used to evaluate the change in survival between the quartiles for UA, and the curves were compared using the log-rank test. A Cox proportional-hazards model, further adjusted in a stepwise manner for age, diabetes, vascular access, albumin, and UA variability, was used. A receiver operating characteristic (ROC) curve was used to evaluate the area under the curves (AUC) to identify UA with statistics used for comparing the different models. Differences with a $p$ value of $<0.05$ (two-tailed) were considered to be statistically significant. The statistical software package $\mathrm{R}$ version 3.6.0 and SPSS for Windows, version 25.0 (SPSS, Chicago, IL, USA), were used for analysis. Graphs were constructed with GraphPad PRISM8 and Photoshop version 6.0.

\section{Results}

A total of 2027 patients were included in the study. According to the inclusion and exclusion criteria, the study eventually included 325 patients undergoing MHD (Figure 1).

Among the 325 patients who were enrolled, 195 patients were men (60\%); 322 (99.1\%) had hypertension. The average age of starting hemodialysis was 57 years, the youngest patient was 20 years old, and the oldest was 88 years old. 


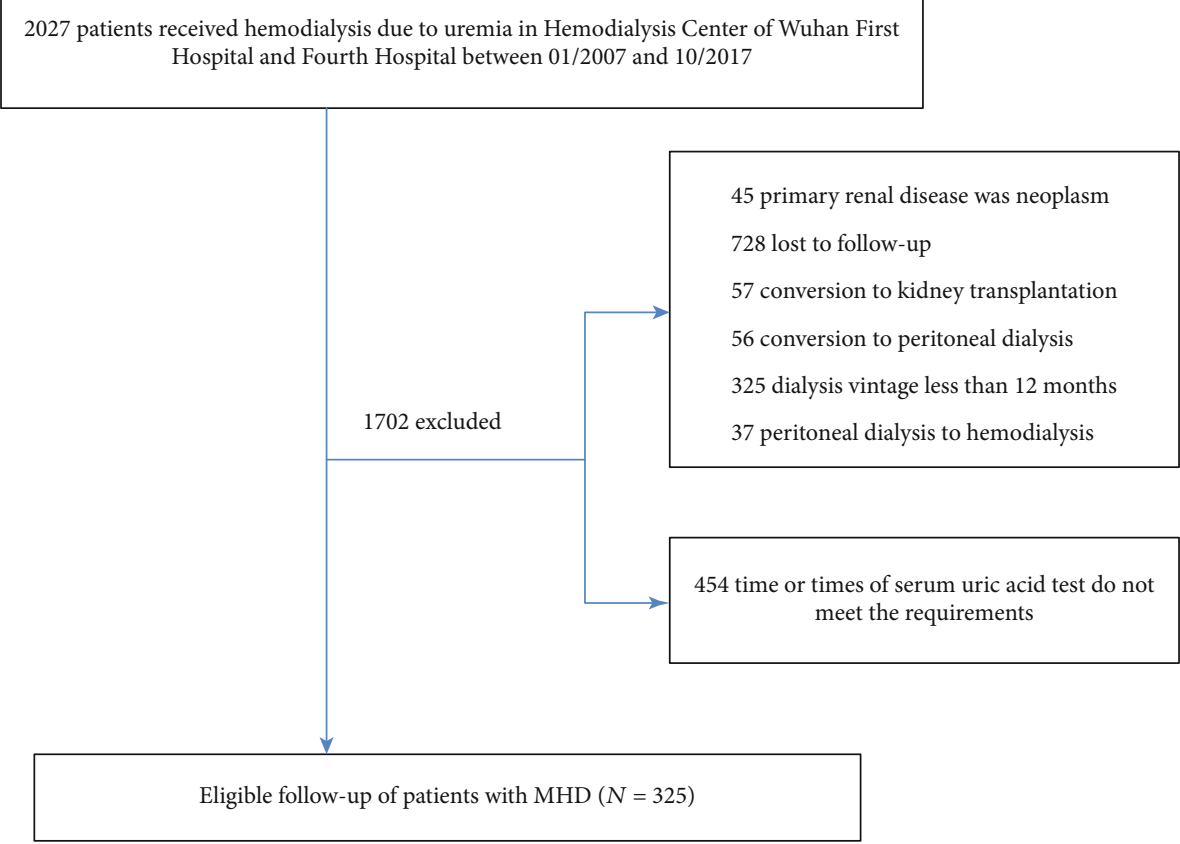

FIgURE 1: The flow chart of population selection. In total, 2027 patients were recruited from the Hemodialysis Center of Wuhan First Hospital and Fourth Hospital. After excluding 1702 participants, the final sample size of 325 participants was enrolled.

The average dialysis vintage was 43 months. There were 236 patients $(72.6 \%)$ who chose arteriovenous fistula (AvF) as the dialysis vascular access, and 89 patients $(27.4 \%)$ were selected for dialysis with tunneled cuffed catheter. The primary disease of uremia included 26 cases $(8 \%)$ who were with hypertensive renal damage, $3(0.9 \%)$ had antineutrophil cytoplasmic antibody- (ANCA-) associated vasculitis with renal damage, 117 (36\%) with primary glomerulonephritis, 147 (45.2\%) with diabetic nephropathy, 11 (3.4\%) with polycystic kidney disease, 3 (0.9\%) with drug-induced renal damage, 10 (3.1\%) with obstructive nephropathy, 6 (1.8\%) with kidney transplant failure, and $2(0.6 \%)$ had lupus nephritis. A total of 116 patients $(35.7 \%)$ died at the end of follow-up; among them, 58 (17.8\%) had cardiac deaths, accounting for $50 \%$ of all-cause deaths, including 24 who had ischemic heart disease, 8 died of malignant arrhythmia, 23 died of congestive heart failure, and 3 who had sudden cardiac death. Other causes of death include infections, gastrointestinal bleeding, multiple organ failure, and cerebrovascular diseases. In the 325 patients, serum UA detection times ranged from 4 to 53. To clarify the effects of different UA levels on all-cause death and cardiac death in patients undergoing hemodialysis, we performed further analysis based on the average serum UA level after quartile grouping.

Baseline clinical data of the four groups of patients are shown in Table 1. Compared with the other three groups, the patients in Quartile 1 were older at the age of starting hemodialysis. Compared with the other two groups, the proportion of AvF as vascular access was significantly higher in Quartile 3 and Quartile 4, at 76.5\% and 82.9\%, respectively. Although patients in all four groups had significantly different mean serum phosphorus and PTH concentrations, the values were still very close. The UA variability was signif- icantly higher in Quartile 4 than in other quartiles. The primary diseases that caused uremia were diverse; however, the two primary diseases were mainly diabetes (147 cases, 45.2\%) and primary glomerulonephritis (117 cases, 36\%). Quartile 1 had the highest occurrence of all-cause death and cardiac death, at $55.6 \%$ and $30.9 \%$, respectively.

We further compared the baseline clinical data between the patients who died and those who survived. The results showed that the age of starting hemodialysis of the death group was significantly higher than that of the surviving group $(66.5 \pm 12.0$ vs. $55.9 \pm 14.7, p<0.001)$. The patients in the death group who used tunneled cuffed catheter were in a higher proportion, and serum phosphorus and PTH levels were better controlled in them than those in the surviving group. The mean serum UA level in the surviving group was significantly higher than that in the death group $(403.3 \pm 72.6$ vs. $364.6 \pm 79.1, p<0.001)$. The primary disease of uremia in the surviving group was mainly primary glomerulonephritis (43.1\%). However, in the death group, it was mainly diabetes (58.6\%), which was much higher than that in the survival group $(37.8 \%)$. We also found that there was no significant difference in UA variability between the two groups $(0.3 \pm 0.125$ vs. $0.286 \pm 0.136, p=0.193)$, as shown in Table 2.

When we did not consider the influence of dialysis vintage, uremic primary disease, and other factors, we found that the risk of all-cause death gradually decreased with the increase of UA among the four groups. Taking Quartile 1 as a reference, the following were observed: Quartile 2, $\mathrm{OR}=0.4$, 95\% CI (0.2-0.8), $p=0.012$; Quartile 3, OR $=0.3,95 \% \mathrm{CI}$ (0.2-0.6), $p<0.001$; and Quartile $4, \mathrm{OR}=0.2,95 \% \mathrm{CI}(0.1-$ $0.5), p<0.001, p$ for trend $<0.001$. The number of all-cause mortalities among the quartiles also gradually decreased; they 
TABLE 1: Characteristics of 325 patients with MHD by serum UA quartiles.

\begin{tabular}{|c|c|c|c|c|c|}
\hline Range of uric acid & $\begin{array}{c}\text { Quartile 1 } \\
183.759-339.491 \\
N=81\end{array}$ & $\begin{array}{c}\text { Quartile } 2 \\
339.491-384.933 \\
N=81\end{array}$ & $\begin{array}{c}\text { Quartile } 3 \\
384.933-438.25 \\
N=81 \\
\end{array}$ & $\begin{array}{c}\text { Quartile } 4 \\
438.25-614.971 \\
N=82 \\
\end{array}$ & $p$ value \\
\hline Age (year) & $63.8 \pm 15.0$ & $58.6 \pm 14.6$ & $59.5 \pm 13.4$ & $57.0 \pm 15.2$ & 0.026 \\
\hline Male (\%) & $41(50.6 \%)$ & $51(63.0 \%)$ & $51(63.0 \%)$ & $52(63.4 \%)$ & \multirow{2}{*}{0.266} \\
\hline Female (\%) & $40(49.4 \%)$ & $30(37.0 \%)$ & $30(37.0 \%)$ & $30(36.6 \%)$ & \\
\hline Dialysis vintage (months) & $39.0 \pm 28.3$ & $44.3 \pm 29.3$ & $45.8 \pm 25.9$ & $44.7 \pm 29.8$ & 0.113 \\
\hline Tunneled cuffed catheter (\%) & $29(35.8 \%)$ & $27(33.3 \%)$ & $19(23.5 \%)$ & $14(17.1 \%)$ & \multirow{2}{*}{0.025} \\
\hline $\operatorname{AvF}(\%)$ & $52(64.2 \%)$ & $54(66.7 \%)$ & $62(76.5 \%)$ & $68(82.9 \%)$ & \\
\hline Hypertension (\%) & $80(98.8 \%)$ & $80(98.8 \%)$ & $80(98.8 \%)$ & $82(100 \%)$ & 0.796 \\
\hline Mean hemoglobin $(g / L)$ & $95.9 \pm 13.7$ & $97.4 \pm 11.1$ & $97.5 \pm 12.8$ & $97.3 \pm 12.1$ & 0.678 \\
\hline Mean phosphorus (mmol/L) & $1.5 \pm 0.4$ & $1.7 \pm 0.3$ & $1.9 \pm 0.4$ & $1.8 \pm 0.4$ & $<0.001$ \\
\hline Mean calcium (mmol/L) & $2.2 \pm 0.1$ & $2.2 \pm 0.1$ & $2.2 \pm 0.1$ & $2.2 \pm 0.1$ & 0.675 \\
\hline Mean PTH $(\mu \mathrm{g} / \mathrm{L})$ & $277.7 \pm 266.9$ & $354.4 \pm 291.3$ & $412.9 \pm 308.3$ & $463.3 \pm 345.2$ & $<0.001$ \\
\hline Mean albumin $(g / L)$ & $36.0 \pm 4.0$ & $37.6 \pm 3.2$ & $38.5 \pm 2.8$ & $39.0 \pm 2.9$ & 0.397 \\
\hline Mean serum uric acid $(\mu \mathrm{mol} / \mathrm{L})$ & $356.9 \pm 72.6$ & $375.7 \pm 65.8$ & $400.0 \pm 75.4$ & $426.7 \pm 77.1$ & $<0.001$ \\
\hline Uric acid variability (\%) & $25.2 \pm 14.5$ & $28.1 \pm 10.7$ & $27.3 \pm 10.7$ & $37.0 \pm 12.3$ & $<0.001$ \\
\hline Primary renal disease $(N)$ & & & & & $<0.001$ \\
\hline Hypertensive kidney lesion & $1(1.2 \%)$ & $8(9.9 \%)$ & $8(9.9 \%)$ & $9(11.0 \%)$ & \\
\hline ANCA & $1(1.2 \%)$ & $0(0.0 \%)$ & $2(2.5 \%)$ & $0(0.0 \%)$ & \\
\hline Diabetes & $57(70.4 \%)$ & $40(49.4 \%)$ & $30(37.0 \%)$ & $20(24.4 \%)$ & \\
\hline Glomerulonephritis & $17(21.0 \%)$ & $24(29.6 \%)$ & $31(38.3 \%)$ & $45(54.9 \%)$ & \\
\hline Drug-induced kidney damage & $1(1.2 \%)$ & $0(0.0 \%)$ & $1(1.2 \%)$ & $1(1.2 \%)$ & \\
\hline Polycystic kidney & $2(2.5 \%)$ & $4(4.9 \%)$ & $2(2.5 \%)$ & $3(3.7 \%)$ & \\
\hline Renal allograft dysfunction & $1(1.2 \%)$ & $1(1.2 \%)$ & $2(2.5 \%)$ & $2(2.4 \%)$ & \\
\hline Lupus nephritis & $0(0.0 \%)$ & $0(0.0 \%)$ & $1(1.2 \%)$ & $1(1.2 \%)$ & \\
\hline Obstructive nephropathy & $1(1.2 \%)$ & $4(4.9 \%)$ & $4(4.9 \%)$ & $1(1.2 \%)$ & \\
\hline \multicolumn{6}{|l|}{ Outcome } \\
\hline All-cause death & $45(55.6 \%)$ & $29(35.8 \%)$ & $23(28.4 \%)$ & $19(23.2 \%)$ & $<0.001$ \\
\hline Cardiac death & $25(30.9 \%)$ & $12(14.8 \%)$ & $10(12.3 \%)$ & $11(13.4 \%)$ & 0.005 \\
\hline
\end{tabular}

$\mathrm{AvF}=$ arteriovenous fistula; $\mathrm{PTH}=$ parathyroid hormone; $N=$ number; $\mathrm{ANCA}=$ antineutrophil cytoplasmic antibodies; $\mathrm{MHD}=$ maintenance hemodialysis; $\mathrm{UA}=$ uric acid.

were $45(55.6 \%), 29(35.8 \%), 23(28.4 \%)$, and 19 patients $(23.2 \%)$ in the four groups, respectively (Figure $2(\mathrm{a}))$. Cardiac death also showed the same trend, and with the increase of UA concentration, the risk of cardiac death was gradually reduced. Taking Quartile 1 as a reference, the following were observed: Quartile 2, OR $=0.4,95 \%$ CI (0.2-0.8), $p=0.017$; Quartile 3, OR = 0.3, 95\% CI (0.1-0.7), $p=0.005$; and Quartile $4, \mathrm{OR}=0.3,95 \%$ CI $(0.2-0.8), p=0.009, p$ for trend $=0.003$. The number of cardiac deaths among the four groups was 25 (30.9\%), 12 (14.8\%), 10 (12.3\%), and 11 (13.4\%), respectively (Figure 2(b)). Among the primary diseases of uremia in the enrolled patients, diabetes accounted for $45.2 \%$ and primary glomerulonephritis for $36 \%$ of them. Therefore, when adjusting for the patient's age, vascular access, diabetes, serum albumin, and UA variability, we found that this trend of influence only existed in all-cause death ( $p$ for trend $=0.001$ ), while it did not exist for cardiac death ( $p$ for trend, 0.0030.101 ) (Table 3). To determine whether there is a difference in UA levels between the death and survival groups, we further compared their UA concentrations. The results showed no significant difference (Figures 3(a) and 3(b)).

As it can be seen that there was no significant difference between Quartile 3 and Quartile 4 in all-cause death and cardiac death, we merged them into Group 3. Group 1 and Group 2 replaced Quartile 1 and Quartile 2, respectively. Then, we performed a single-factor Kaplan-Meier survival analysis. The results show that the median survival time for all-cause mortality (40 months, 95\% CI, 33.261-46.739) and cardiac death (69 months, 95\% CI, 43.608-94.392) in Group 1 was much lower than that in Group 2 and Group 3 (Logrank $p<0.001)$, but the difference between Group 2 and Group 3 was not significant $(p=0.121$ and $p=0.701$, respectively) (Figure 4). The univariate Cox regression analysis showed that the age of starting hemodialysis, diabetes, serum albumin, vascular access and UA concentration and its variability interaction were risk factors for all-cause mortality. However, when adjusting for confounding factors, we found that an increase in UA levels reduced the risk of all-cause 
TABLE 2: Characteristics of alive and dead patients.

\begin{tabular}{|c|c|c|c|}
\hline Status & Alive & Dead & $p$ value \\
\hline Number of patients & $n=209$ & $n=116$ & \\
\hline Range of uric acid variability values (\%) & $30 \pm 12.5$ & $28.6 \pm 13.6$ & 0.193 \\
\hline Age (year) & $55.9 \pm 14.7$ & $66.5 \pm 12.0$ & $<0.001$ \\
\hline Male & $124(59.3 \%)$ & $71(61.2 \%)$ & \multirow{2}{*}{0.741} \\
\hline Female & $85(40.7 \%)$ & $45(38.8 \%)$ & \\
\hline Tunneled cuffed catheter (\%) & $50(23.9 \%)$ & $39(33.6 \%)$ & \multirow{2}{*}{0.06} \\
\hline $\operatorname{AvF}(\%)$ & $159(76.1 \%)$ & $77(66.4 \%)$ & \\
\hline Hypertension (\%) & $207(99.0 \%)$ & $115(99.1 \%)$ & 0.932 \\
\hline Hemoglobin (g/L) & $97.8 \pm 12.4$ & $95.7 \pm 12.4$ & 0.132 \\
\hline Phosphorus (mmol/L) & $1.8 \pm 0.4$ & $1.6 \pm 0.4$ & $<0.001$ \\
\hline Calcium $(\mathrm{mmol} / \mathrm{L})$ & $2.2 \pm 0.2$ & $2.2 \pm 0.1$ & 0.904 \\
\hline PTH $(\mu \mathrm{g} / \mathrm{L})$ & $428.4 \pm 334.9$ & $277.9 \pm 225.0$ & $<0.001$ \\
\hline Albumin $(\mathrm{g} / \mathrm{L})$ & $38.2 \pm 3.1$ & $36.8 \pm 3.8$ & $<0.001$ \\
\hline Uric acid $(\mu \mathrm{mol} / \mathrm{L})$ & $403.3 \pm 72.6$ & $364.6 \pm 79.1$ & $<0.001$ \\
\hline Primary renal disease $(N)$ & & & 0.003 \\
\hline Hypertensive kidney lesion & $16(7.7 \%)$ & $10(8.6 \%)$ & \\
\hline ANCA & $1(0.5 \%)$ & $2(1.7 \%)$ & \\
\hline Diabetes & $79(37.8 \%)$ & $68(58.6 \%)$ & \\
\hline Glomerulonephritis & $90(43.1 \%)$ & $27(23.3 \%)$ & \\
\hline Drug-induced kidney damage & $3(1.4 \%)$ & $0(0.0 \%)$ & \\
\hline Polycystic kidney & $7(3.3 \%)$ & $4(3.4 \%)$ & \\
\hline Renal allograft dysfunction & $6(2.9 \%)$ & $0(0.0 \%)$ & \\
\hline Lupus nephritis & $2(1.0 \%)$ & $0(0.0 \%)$ & \\
\hline Obstructive nephropathy & $5(2.4 \%)$ & $5(4.3 \%)$ & \\
\hline
\end{tabular}

$\mathrm{AvF}=$ arteriovenous fistula; $\mathrm{PTH}=$ parathyroid hormone; $N=$ number; $\mathrm{ANCA}=$ antineutrophil cytoplasmic antibodies.

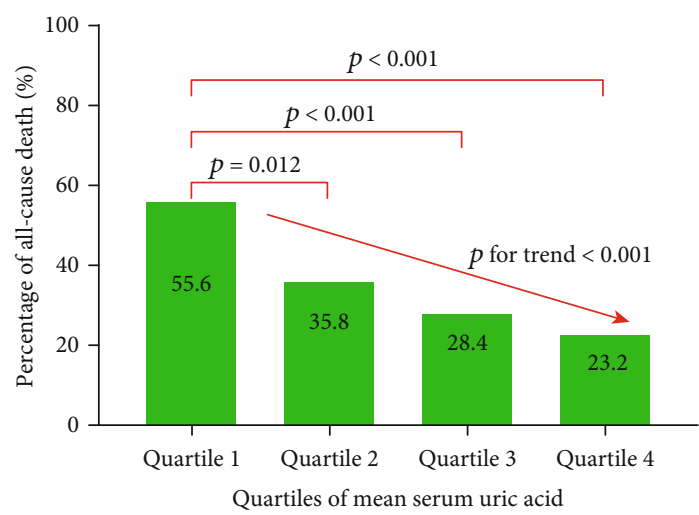

(a)

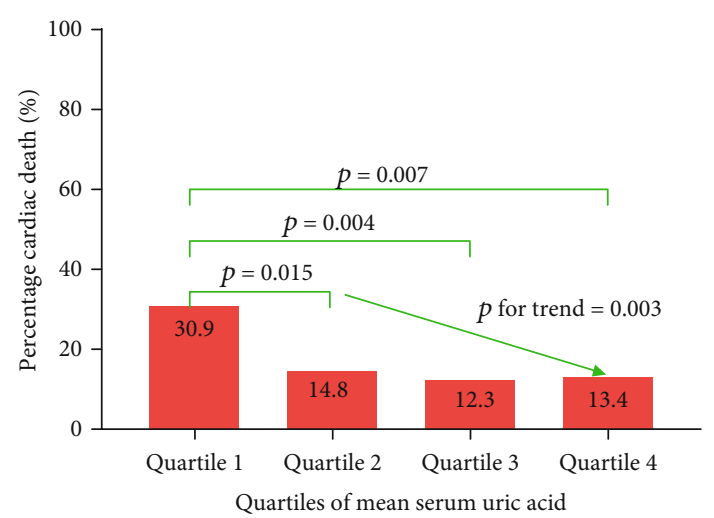

(b)

FIgure 2: Mean serum uric acid (UA) and rates of (a) all-cause death and (b) cardiac death. Quartile 1, Quartile 2, Quartile 3, and Quartile 4 are quantile grouping based on UA levels.

mortality, but the interaction between UA and variability no longer had this characteristic. In contrast, after adjusting for multiple confounding factors, both serum UA concentration and interaction between UA and its variability were not associated with cardiac death, as shown in Table 4.

To determine the predictive effect of the UA level and its variability on all-cause mortality and cardiac death in patients undergoing MHD, we used the ROC for analysis. The results showed that serum UA predicted all-cause mortality, the AUC was 0.6593 (95\% CI, 0.596-0.7225), specificity was $78.95 \%$, and sensitivity was $46.96 \%$, while for cardiac death, AUC was 0.6376 (95\% CI, 0.5529-0.7223). The variability of UA had less predictive value in all-cause mortality and cardiac death. Their AUC were 0.5437 and 0.4451 , respectively. We 
TABLE 3: The association between mean uric acid levels in hemodialysis patients and the risk of all-cause mortality.

\begin{tabular}{|c|c|c|c|c|c|}
\hline \multicolumn{6}{|c|}{ The association between mean uric acid levels in hemodialysis patients and the risk of all-cause mortality } \\
\hline Variable & $\begin{array}{c}\text { Quartile 1 } \\
\text { (183.759-339.491 } \mu \mathrm{mol} / \mathrm{L}) \\
\text { Reference }\end{array}$ & $\begin{array}{c}\text { Quartile } 2 \\
(339.491-384.933 \mu \mathrm{mol} / \mathrm{L}) \\
\text { OR }(95 \% \mathrm{CI})\end{array}$ & $\begin{array}{c}\text { Quartile } 3 \\
(384.933-438.25 \mu \mathrm{mol} / \mathrm{L}) \\
\text { OR }(95 \% \mathrm{CI})\end{array}$ & $\begin{array}{c}\text { Quartile } 4 \\
(438.25-614.971 \mu \mathrm{mol} / \mathrm{L}) \\
\text { OR }(95 \% \mathrm{CI})\end{array}$ & $p$ value for trend \\
\hline Model 1 & 1 & $0.4(0.2-0.8)$ & $0.3(0.2-0.6)$ & $0.2(0.1-0.5)$ & $<0.001$ \\
\hline Model 2 & 1 & $0.6(0.3-1.1)$ & $0.4(0.2-0.7)$ & $0.3(0.1-0.7)$ & 0.001 \\
\hline \multicolumn{6}{|c|}{ The association between mean uric acid levels in hemodialysis patients and the risk of cardiac death } \\
\hline Variable & $\begin{array}{c}\text { Quartile 1 } \\
(183.759-339.491 \mu \mathrm{mol} / \mathrm{L}) \\
\text { Reference }\end{array}$ & $\begin{array}{c}\text { Quartile } 2 \\
(339.491-384.933 \mu \mathrm{mol} / \mathrm{L}) \\
\text { OR }(95 \% \mathrm{CI})\end{array}$ & $\begin{array}{c}\text { Quartile } 3 \\
(384.933-438.25 \mu \mathrm{mol} / \mathrm{L}) \\
\text { OR }(95 \% \mathrm{CI})\end{array}$ & $\begin{array}{c}\text { Quartile } 4 \\
(438.25-614.971 \mu \mathrm{mol} / \mathrm{L}) \\
\text { OR }(95 \% \mathrm{CI})\end{array}$ & $p$ value for trend \\
\hline Model 1 & 1 & $0.4(0.2-0.8)$ & $0.3(0.1-0.7)$ & $0.3(0.2-0.8)$ & 0.003 \\
\hline Model 2 & 1 & $0.5(0.2-1.2)$ & $0.4(0.2-1.0)$ & $0.6(0.2-1.4)$ & 0.101 \\
\hline
\end{tabular}

Model 1: unadjusted. Model 2: adjusted for age, vascular access, albumin, diabetes, and uric acid variability.

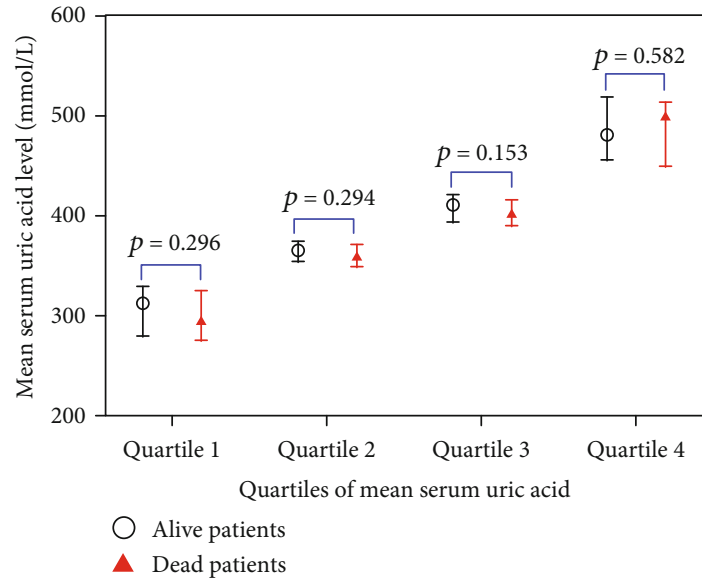

(a)

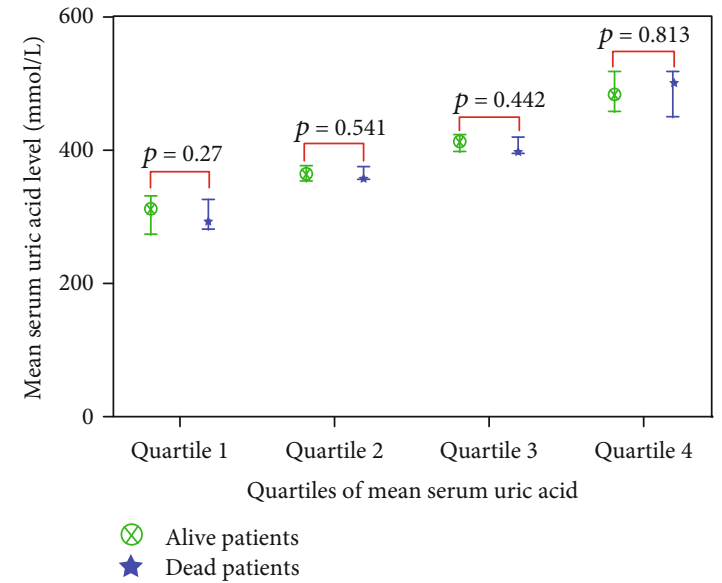

(b)

FIGURE 3: Comparing the differences in UA between dead and surviving patients in each group. Quartile 1, Quartile 2, Quartile 3, and Quartile 4 are quantile grouping based on UA levels.

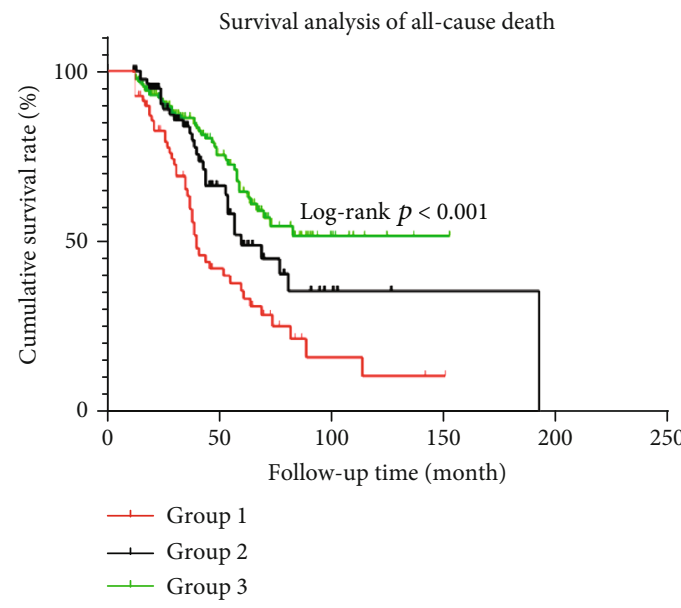

(a)

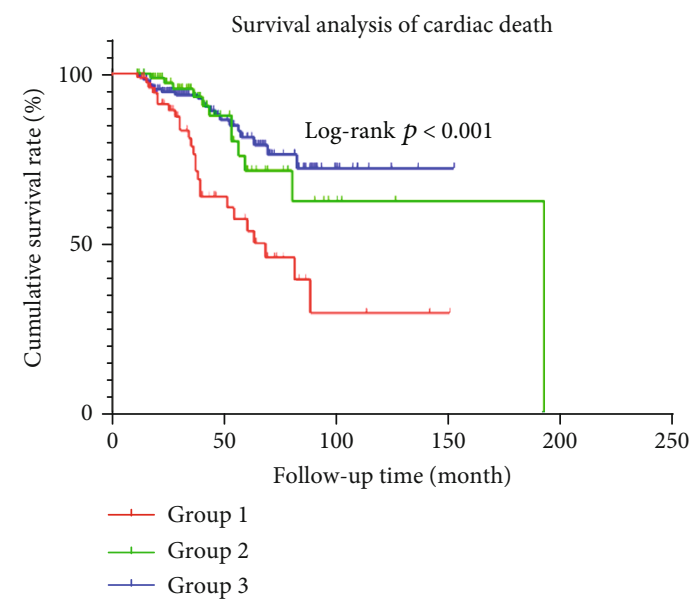

(b)

FIGURE 4: Kaplan-Meier curves for survival free of (a) all-cause death and (b) cardiac death stratified by groups based on the UA levels. 
TABLE 4: Cox regression analysis of all-cause mortality and cardiac death.

\begin{tabular}{|c|c|c|c|c|}
\hline \multirow{2}{*}{ Variable } & \multicolumn{2}{|c|}{ Univariable } & \multicolumn{2}{|c|}{ Multivariable } \\
\hline & $\operatorname{HR}(95 \% \mathrm{CI})$ & $p$ value & $\operatorname{HR}(95 \% \mathrm{CI})$ & $p$ value \\
\hline \multicolumn{5}{|c|}{ Cox regression analysis of all-cause mortality } \\
\hline Age (years) & $1.058(1.042-1.074)$ & $<0.001$ & $1.044(1.026-1.062)$ & $<0.001$ \\
\hline Diabetes $($ yes $=1$, no $=0$ ) & $2.154(1.477-3.14)$ & $<0.001$ & $1.751(1.138-2.696)$ & 0.011 \\
\hline Vascular access $(1=$ catheter, $2=\mathrm{AvF})$ & $0.406(0.274-0.602)$ & $<0.001$ & $0.759(0.484-1.189)$ & 0.228 \\
\hline Albumin $(\mathrm{g} / \mathrm{L})$ & $0.819(0.776-0.865)$ & $<0.001$ & $0.899(0.842-0.96)$ & 0.001 \\
\hline Mean uric acid $(\mu \mathrm{mol} / \mathrm{L})$ & $0.995(0.992-0.997)$ & $<0.001$ & $0.996(0.992-1.000)$ & 0.035 \\
\hline Mean uric acid* variability & $1.0(1.0-1.0)$ & 0.042 & $1.0(1.0-1.0)$ & 0.223 \\
\hline \multicolumn{5}{|l|}{ Cox regression analysis of cardiac death } \\
\hline Age (years) & $1.073(1.049-1.098)$ & $<0.001$ & $1.066(1.037-1.096)$ & $<0.001$ \\
\hline Diabetes $($ yes $=1$, no $=0$ ) & $3.157(1.788-5.572)$ & $<0.001$ & $3.114(1.577-6.150)$ & 0.001 \\
\hline Vascular access $(1=$ catheter, $2=\mathrm{AvF})$ & $0.322(0.187-0.554)$ & $<0.001$ & $0.605(0.324-1.130)$ & 0.115 \\
\hline Albumin $(\mathrm{g} / \mathrm{L})$ & $0.835(0.77-0.905)$ & $<0.001$ & $0.957(0.869-1.054)$ & 0.369 \\
\hline Mean uric acid $(\mu \mathrm{mol} / \mathrm{L})$ & $0.994(0.99-0.998)$ & 0.001 & $0.994(0.988-1.0)$ & 0.056 \\
\hline Mean uric acid* variability & $1.0(1.0-1.0)$ & 0.146 & $1.0(1.0-1.0)$ & 0.098 \\
\hline
\end{tabular}

HR (hazard ratio) indicates the relative increased risk of all-cause or cardiac death with each change in age, mean uric acid, uric acid variability, albumin, diabetes positive, or vascular access. Mean uric acid*variability reflected the interaction between uric acid and its variability. In multivariable regression analysis, these six variables were included in the same model.

found that the age of starting hemodialysis was an independent risk factor for all-cause mortality and cardiovascular death in patients undergoing MHD in both univariate and multivariate analyses. Therefore, we introduced it to predict all-cause death and cardiac death, and we found that there was no significant difference in the predictive value between age and UA (ROC-AUC ratio was 0.7134 vs. $0.6593, p=0.2009 ; 0.7124$ vs. $0.6376, p=0.1888$ ). However, when age was combined with UA and its variability, the diagnostic efficacy could be significantly improved. The AUC value for diagnosis of all-cause mortality was increased to 0.7517 (95\% CI, 0.6969-0.8064, $p=0.0245$ ), and the sensitivity was increased to $71.93 \%$. The AUC value for diagnosis of cardiac death was increased to 0.7394 (95\% CI, 0.6677 $0.811, p=0.2194)$ and the sensitivity was increased to $75 \%$ (Figures 5(a) and 5(b)).

\section{Discussion}

This article retrospectively analyzed the relationship between serum UA levels and its fluctuations in patients in terms of all-cause mortality and cardiac death. After adjusting for confounding factors, we found that long-term low-level UA $(<339.491 \mu \mathrm{mol} / \mathrm{L})$ significantly increased the risk of allcause mortality. However, the range of variation in UA levels could affect the occurrence of cardiovascular events in patients, which was not clear at this time. We also found that the higher age of starting hemodialysis of patients with serum UA levels increased the predictive value of long-term prognosis in these patients.

UA is the terminal metabolite of purine, and $80 \%$ of the total UA in the human body is produced by the catabolism of nuclear proteins and $20 \%$ produced by food which contains more purine. UA is basically present in the blood in the free form as monosodium urate. About $70 \%$ of UA is cleared by the kidneys, and the remaining $30 \%$ is cleared by the gastrointestinal tract [18]. Researchers had found that raised serum UA levels were significantly associated with a rapid decline in renal function and the incidence of $\mathrm{CKD}$. Therefore, patients with chronic renal failure often develop hyperuricemia, especially in patients with advanced renal failure [19]. The biological role of UA in the human body is mainly reflected in two aspects. Firstly, the combination of UA with ammonia and urea plays an important role in the removal of nitrogen-containing compounds [20]. Secondly, $\mathrm{UA}$ is also an antioxidant. It can interact with hydrogen peroxide and hydroxyl radicals to effectively scavenge free radicals in the body, thus protecting vascular endothelial cells [21]. At present, there is no unified consensus on the impact of UA on the prognosis of patients undergoing hemodialysis. Some studies suggest that the raised UA levels increase the risk of death in hemodialysis or peritoneal dialysis patients $[13,14,22]$. Some researchers have also found that lower levels of UA increase cardiac death and all-cause death in these patients $[23,24]$. Moreover, studies have shown that as time progresses, longitudinal increases in UA reduce allcause mortality and cardiac death in these patients [25]. These follow-up studies were based on average UA or a certain baseline level of UA.

However, since the patient's UA level varies, the question remains whether the changed UA also affects the patient's prognosis. There is no similar study at the moment. We found that the UA variability has no effect on all-cause death and cardiac death. The patient's mean serum UA level was associated with prognosis. Without considering the confounding factors, all-cause death and cardiac death gradually decreased with the increase of UA, which is consistent with previous studies [25-27]. After adjusting for the confounding factors, the results of multivariate Cox regression analysis showed that with the increase of UA, all-cause death of patients still 


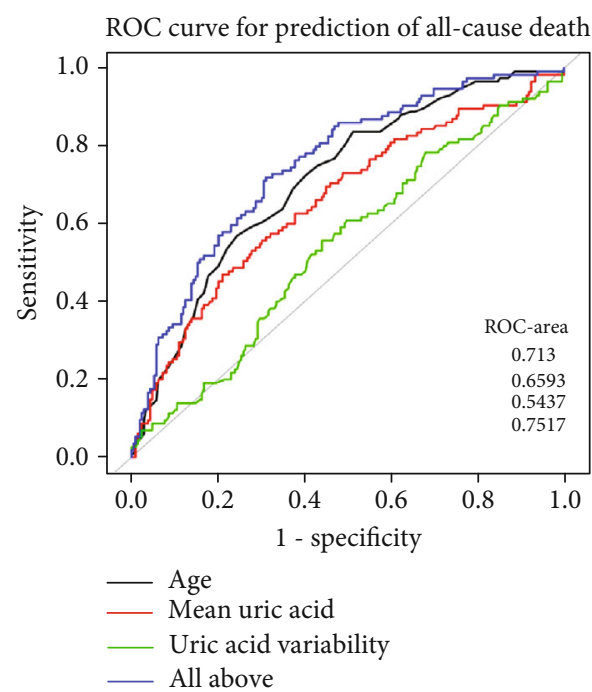

(a)

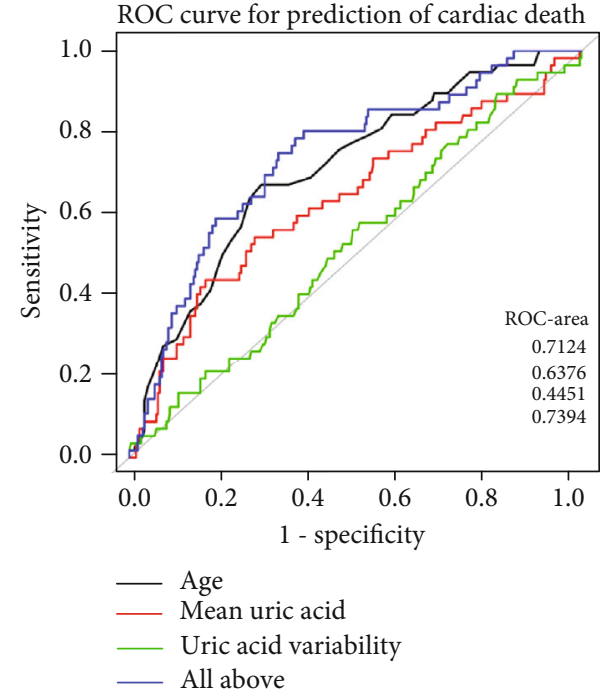

(b)

FIgURE 5: (a) Receiver operating characteristic curve (ROC) analysis for the prediction of all-cause death. Model 1 (black) includes age; Model 2 (red) includes UA; Model 3 (green) includes UA variability; and Model 4 (blue) includes age and UA and its variability. (b) ROC analysis for the prediction of cardiac death. Model 1 (black) includes age; Model 2 (red) includes UA; Model 3 (green) includes UA variability; and Model 4 (blue) includes age and UA and its variability.

gradually decreased, but the impact on cardiac death was no longer obvious ( $p=0.001, p=0.058)$. Chang et al. and others recorded similar findings $[24,28]$. We also found that average UA levels below $339.491 \mu \mathrm{mol} / \mathrm{L}$ were an independent risk factor for all-cause mortality; this finding was consistent with that of a prospective cohort study by Korean researchers. Their study showed that a mean UA of $<5.5 \mathrm{mg} / \mathrm{dL}$ in patients undergoing MHD was independently associated with allcause mortality [29].

The mechanism of UA by which its level affects all-cause mortality and cardiovascular death in hemodialysis patients was unclear. It could essentially be the result of the comprehensive influence of many factors. High-risk deaths due to low UA levels may be related to the following causes. Studies have shown that UA levels were positively correlated with albumin and negatively correlated with the Charlson comorbidity index [30]. Thus, the risk of high mortality due to low UA levels might be because of bad nutritional status associated with hypoalbuminemia, and heavier comorbidities led to higher risk of death. Secondly, it could also be that excessive oxidative stress caused by low UA levels, and by inducing endothelial dysfunction, indirectly led to a higher risk of death $[31,32]$.

Our research found that whether it was single factor or multifactor analysis, the risk of all-cause death or cardiac death increased with age, which was consistent with previous studies $[33,34]$. We used the ROC curve to analyze the predictive value of the age of starting hemodialysis for longterm prognosis in patients undergoing MHD. The results showed that there was no apparent difference in statistical efficacy compared with UA, but the combined predictive value of the two was significantly improved.

This study had several limitations. (1) This was a retrospective cohort study with a small sample size, which may have led to a bias. (2) The primary diagnosis of uremia in some patients was based on clinical diagnosis and not pathological diagnosis. (3) As we only collected data from patients at baseline, such as calcium, phosphorus, and PTH, we were unable to analyze the impact of these baseline changes on patient outcomes during follow-up. Therefore, we failed to correct the traditional risk factors for uremia and cardiovascular events during multivariate Cox regression analysis. (4) The oral medications commonly used by patients, cholesterol and $\mathrm{Kt} / \mathrm{v}$ data, were not included in the analysis. (5) This study only specified the lower limit of UA level in patients undergoing MHD. Thus, the extent to which UA elevation requires intervention remains unclear.

In summary, through this retrospective cohort study, we showed that low UA levels are closely related to all-cause mortality in patients undergoing MHD. However, they have no significant effect on cardiac death. Moreover, they have a good predictive value for the long-term prognosis of such patients.

\section{Data Availability}

The data used to support the findings of this study are included within the article.

\section{Conflicts of Interest}

The authors declare that they have no conflicts of interest.

\section{Authors' Contributions}

Ze-Xuan Dong and Ming Tian are equal contributors. 


\section{Acknowledgments}

This study was funded by the Hubei Province Natural Science Fund (2014CFC1047).

\section{References}

[1] Y. Zhu, B. J. Pandya, and H. K. Choi, "Prevalence of gout and hyperuricemia in the US general population: the National Health and Nutrition Examination Survey 2007-2008," Arthritis and Rheumatism, vol. 63, no. 10, pp. 3136-3141, 2011.

[2] E. P. de Oliveira and R. C. Burini, "High plasma uric acid concentration: causes and consequences," Diabetology \& Metabolic Syndrome, vol. 4, no. 1, p. 12, 2012.

[3] R. Horikoshi, T. Akimoto, M. Inoue, Y. Morishita, and E. Kusano, "Febuxostat for hyperuricemia: experience with patients on chronic hemodialysis treatment," Clinical and Experimental Nephrology, vol. 17, no. 1, pp. 149-150, 2013.

[4] N. Tangri and D. E. Erlich, "Uric acid, CKD, and cardiovascular disease: confounders, culprits, and circles," American Journal of Kidney Diseases the Official Journal of the National Kidney Foundation, vol. 56, no. 2, pp. 247-250, 2010.

[5] W. Karwowski, B. Naumnik, M. Szczepański, and M. Myśliwiec, "The mechanism of vascular calcification - a systematic review," Medical Science Monitor, vol. 18, no. 1, pp. RA1-RA11, 2012.

[6] C. T. Lee, S. Chua, C. Y. Hsu et al., "Biomarkers associated with vascular and valvular calcification in chronic hemodialysis patients," Disease Markers, vol. 34, no. 4, pp. 229-235, 2013.

[7] B. Ekici, U. Kütük, A. Alhan, and H. F. Töre, "The relationship between serum uric acid levels and angiographic severity of coronary heart disease," Kardiologia Polska, vol. 73, no. 7, pp. 533-538, 2015.

[8] M. Li, X. Hu, Y. Fan et al., "Hyperuricemia and the risk for coronary heart disease morbidity and mortality a systematic review and dose-response meta-analysis," Scientific Reports, vol. 6, no. 1, article 19520, 2016.

[9] K. Lottmann, X. Chen, and P. K. Schädlich, “Association between gout and all-cause as well as cardiovascular mortality: a systematic review," Current Rheumatology Reports, vol. 14, no. 2, pp. 195-203, 2012.

[10] A. H. Wu, J. D. Gladden, M. Ahmed, A. Ahmed, and G. Filippatos, "Relation of serum uric acid to cardiovascular disease," International Journal of Cardiology, vol. 213, pp. 47, 2016.

[11] C. Borghi, A. Tykarski, K. Widecka et al., "Expert consensus for the diagnosis and treatment of patient with hyperuricemia and high cardiovascular risk," Cardiology Journal, vol. 25, no. 5, pp. 545-563, 2018.

[12] M. E. Suliman, R. J. Johnson, E. García-López et al., "J-shaped mortality relationship for uric acid in CKD," American Journal of Kidney Diseases, vol. 48, no. 5, pp. 761-771, 2006.

[13] J. S. Jeon, S. H. Chung, D. C. Han et al., "Mortality predictive role of serum uric acid in diabetic hemodialysis patients," Journal of Renal Nutrition, vol. 24, no. 5, pp. 336-342, 2014.

[14] X. Xia, F. He, X. Wu, F. Peng, F. Huang, and X. Yu, "Relationship between serum uric acid and all-cause and cardiovascular mortality in patients treated with peritoneal dialysis," American Journal of Kidney Diseases, vol. 64, no. 2, pp. 257-264, 2014.
[15] W. Chang, W. Zhang, X. Wang et al., "The association of longitudinal serum uric acid and all-cause mortality in incident peritoneal dialysis patients," Blood Purification, vol. 47, no. 1-3, pp. 185-192, 2018.

[16] A. Gouri, A. Dekaken, A. A. Bentorki, A. Touaref, A. Yakhlef, and N. Kouicem, "Serum uric acid level and cardiovascular risks in hemodialysis patients: an algerian cohort study," Clinical Laboratory, vol. 60, no. 5, pp. 751-758, 2014.

[17] M. C. Bouzas-Mosquera, A. Bouzas-Mosquera, and J. Peteiro, "Excessive blood pressure increase with exercise and risk of all-cause mortality and cardiac events," European Journal of Clinical Investigation, vol. 46, no. 10, pp. 833-839, 2016.

[18] P. Dousdampanis, "The grey zone of hyperuricemia in chronic kidney disease," Journal of Advanced Research, vol. 8, no. 5, pp. 549-550, 2017.

[19] S. E. Mwasongwe, T. Fülöp, R. Katz et al., "Relation of uric acid level to rapid kidney function decline and development of kidney disease: the Jackson Heart Study," Journal of Clinical Hypertension, vol. 20, no. 4, pp. 775-783, 2018.

[20] P. A. Wright, "Nitrogen excretion: three end products, many physiological roles," Journal of Experimental Biology, vol. 198, Part 2, pp. 273-281, 1995.

[21] M. Kanbay, M. Segal, B. Afsar, D. H. Kang, B. Rodriguez-Iturbe, and R. J. Johnson, "The role of uric acid in the pathogenesis of human cardiovascular disease," Heart, vol. 99, no. 11, pp. 759766, 2013.

[22] T. Petreski, R. Ekart, R. Hojs, and S. Bevc, "Asymptomatic hyperuricemia and cardiovascular mortality in patients with chronic kidney disease who progress to hemodialysis," International Urology and Nephrology, vol. 51, no. 6, pp. 10131018, 2019.

[23] W. Latif, A. Karaboyas, L. Tong et al., "Uric acid levels and allcause and cardiovascular mortality in the hemodialysis population," Clinical Journal of the American Society of Nephrology, vol. 6, no. 10, pp. 2470-2477, 2011.

[24] C. Park, Y. Obi, E. Streja et al., "Serum uric acid, protein intake and mortality in hemodialysis patients," Nephrology, Dialysis, Transplantation, vol. 32, no. 10, article 1750, 2017.

[25] I. Beberashvili, A. Erlich, A. Azar et al., "Longitudinal study of serum uric acid, nutritional status, and mortality in maintenance hemodialysis patients," Clinical Journal of the American Society of Nephrology, vol. 11, no. 6, pp. 1015-1023, 2016.

[26] I. Beberashvili, I. Sinuani, A. Azar et al., "Serum uric acid as a clinically useful nutritional marker and predictor of outcome in maintenance hemodialysis patients," Nutrition, vol. 31, no. 1, pp. 138-147, 2015.

[27] N. Sugano, Y. Maruyama, S. Kidoguchi et al., "Effect of hyperuricemia and treatment for hyperuricemia in Japanese hemodialysis patients: a cohort study," PLoS One, vol. 14, no. 6, article e0217859, 2019.

[28] C. S. Kim, D. C. Jin, Y. C. Yun, E. H. Bae, S. K. Ma, and S. W. Kim, "Relationship between serum uric acid and mortality among hemodialysis patients: retrospective analysis of Korean end-stage renal disease registry data," Kidney Research and Clinical Practice, vol. 36, no. 4, pp. 368-376, 2017.

[29] E. Bae, H. J. Cho, N. Shin et al., "Lower serum uric acid level predicts mortality in dialysis patients," Medicine, vol. 95, no. 24, article e3701, 2016.

[30] S. M. K. Lee, A. L. Lee, T. J. Winters et al., "Low serum uric acid level is a risk factor for death in incident hemodialysis 
patients," American Journal of Nephrology, vol. 29, no. 2, pp. 79-85, 2009.

[31] B. De Becker, C. Coremans, M. Chaumont et al., "Severe hypouricemia impairs endothelium-dependent vasodilatation and reduces blood pressure in healthy young men: A randomized, placebo-controlled, and crossover study," Journal of the American Heart Association, vol. 8, no. 23, p. e013130, 2019.

[32] M. Murea and B. M. Tucker, "The physiology of uric acid and the impact of end-stage kidney disease and dialysis," Seminars in Dialysis, vol. 32, no. 1, pp. 47-57, 2019.

[33] A. Kramer, M. Pippias, V. S. Stel et al., "Renal replacement therapy in Europe: a summary of the 2013 ERA-EDTA Registry Annual Report with a focus on diabetes mellitus," Clinical Kidney Journal, vol. 9, no. 3, pp. 457-469, 2016.

[34] I. M. Ivana, I. Bubic, B. Sladoje-Martinovic, S. Racki, and L. Orlic, "Survival of chronic hemodialysis patients over 80 years of age," Clinical Interventions in Aging, vol. 9, no. 2, pp. 689-696, 2014. 